Elect. Comm. in Probab. 13 (2008), 526-539

ELECTRONIC

COMMUNICATIONS

in PROBABILITY

\title{
FREE GENERALIZED GAMMA CONVOLUTIONS
}

\author{
VICTOR PÉREZ-ABREU ${ }^{1}$ \\ Department of Probability and Statistics, CIMAT, Apdo. Postal 402, Guanajuato, Gto. 36000, México \\ email: pabreu@cimat.mx \\ NORIYOSHI SAKUMA ${ }^{2}$ \\ Department of Mathematics, Keio University, 3-14-1 Hiyoshi, Kohoku-ku, Yokohama, 223-8522, \\ Japan \\ email: noriyosi@math.keio.ac.jp
}

Submitted May 14, 2008, accepted in final form September 8, 2008

AMS 2000 Subject classification: 15A52, 46L54, 60E07

Keywords: free probability, infinitely divisible distribution, generalized gamma convolutions, Random matrices

Abstract

The so-called Bercovici-Pata bijection maps the set of classical infinitely divisible laws to the set of free infinitely divisible laws. The purpose of this work is to study the free infinitely divisible laws corresponding to the classical Generalized Gamma Convolutions (GGC). Characterizations of their free cumulant transforms are derived as well as free integral representations with respect to the free Gamma process. A random matrix model for free GGC is built consisting of matrix random integrals with respect to a classical matrix Gamma process. Nested subclasses of free GGC are shown to converge to the free stable class of distributions.

\section{Introduction}

Generalized Gamma Convolutions (GGC) is the smallest class $T^{*}\left(\mathbb{R}_{+}\right)$of classical infinitely divisible distributions on $\mathbb{R}_{+}$that contains all Gamma distributions and that is closed under classical convolution and weak convergence. This class was introduced by Thorin [16], [17] and further studied by Bondesson [7]. Thorin [18] also considered the smallest class of distributions on the real line which contains all distributions in $T^{*}\left(\mathbb{R}_{+}\right)$and is closed under convolution, convergence and reflection. We denote this class by $T^{*}(\mathbb{R})$ and called it the Thorin class of distributions on $\mathbb{R}$. Let $\mathscr{P}(\mathbb{R})$ be set of probability measures on $\mathbb{R}$ and $I^{*}(\mathbb{R})$ the class of all classical infinitely divisible distributions in $\mathscr{P}(\mathbb{R})$. If $\mu \in \mathscr{P}(\mathbb{R}), \hat{\mu}(z)$ denotes its Fourier transform and when $\mu \in I^{*}(\mathbb{R})$

\footnotetext{
${ }^{1}$ PART OF THIS WORK WAS DONE WHILE THE AUTHOR WAS VISITING KEIO UNIVERSITY DURING THE FALL OF 2007. HE ACKNOWLEDGES THE SUPPORT AND HOSPITALITY OF THE MATHEMATICS DEPARTMENT OF THIS UNIVERSITY

${ }^{2}$ PART OF THIS WORK WAS DONE WHILE THE AUTHOR WAS VISITING CIMAT DURING THE SPRING OF 2008. HE SINCERELY APPRECIATES THE SUPPORT AND HOSPITALITY OF CIMAT. HE IS SUPPORTED BY JAPAN SOCIETY FOR THE PROMOTION OF SCIENCE.
} 
we denote by $\mathscr{C}_{\mu}^{*}(z)$ its classical cumulant function or Lévy exponent i.e. $\mathscr{C}_{\mu}^{*}(z)=\log \hat{\mu}(z)$. A probability measure $\mu \in \mathscr{P}(\mathbb{R})$ is in $I^{*}(\mathbb{R})$ if and only if its classical cumulant function has the Lévy-Khintchine representation :

$$
\mathscr{C}_{\mu}^{*}(z)=-\frac{1}{2} a z^{2}+i \eta z+\int_{\mathbb{R}}\left(e^{-i z x}-1-i z x 1_{\{|x| \leq 1\}}\right) v(d x), \quad z \in \mathbb{R},
$$

where $a \geq 0, \gamma \in \mathbb{R}$ and $v$ (the so called Lévy measure) is a measure satisfying $v(\{0\})=0$ and $\int_{\mathbb{R}}\left(1 \wedge|x|^{2}\right)<\infty$. The triplet $(a, \eta, v)$ is uniquely determined and is called $*$-characteristic triplet or simply $*$-triplet. When $\int_{\mathbb{R}}|x| v(d x)<\infty$, we speak of the drift type Lévy Khintchine representation

$$
\mathscr{C}_{\mu}^{*}(z)=-\frac{1}{2} a z^{2}+i \eta^{\prime} z+\int_{\mathbb{R}}\left(e^{-i z x}-1\right) v(d x)(z \in \mathbb{R}),
$$

where $\eta^{\prime}$ is the drift of $\mu$ and is given by $\eta^{\prime}=\eta-\int_{\{|x| \leq 1\}} x v(d x)$. We write

$$
I_{\log }^{*}(\mathbb{R})=\left\{\mu \in I^{*}(\mathbb{R}) ; \int_{\mathbb{R}} \log (|x| \wedge 1) \mu(\mathrm{d} x)<\infty\right\}
$$

and refer to Sato [13] for basic facts about classical infinitely divisible distributions.

Bondesson [7] proved that a positive random variable $Y$ with classical law $\mathscr{L}^{*}(Y)=\mu$-without translation term- belongs to $T^{*}\left(\mathbb{R}_{+}\right)$if and only if there exists a positive Radon measure $U_{\mu}$ on $(0, \infty)$ such that

$$
\begin{aligned}
\mathscr{C}_{\mu}^{*}(z) & =-\int_{0}^{\infty}\left(1-e^{i z x}\right) \frac{\mathrm{d} x}{x} \int_{0}^{\infty} e^{-x s} U_{\mu}(\mathrm{d} s) \\
& =-\int_{0}^{\infty} \log \left(1+\frac{i z}{s}\right) U_{\mu}(\mathrm{d} s)
\end{aligned}
$$

with $\int_{0}^{1}|\log x| U_{\mu}(\mathrm{d} x)<\infty$ and $\int_{1}^{\infty} \frac{U_{\mu}(\mathrm{d} x)}{x}<\infty$. The measure $U_{\mu}$ is called the Thorin measure of $\mu$. So, the $*$-triplet of $\mu$ is $\left(0,0, v_{\mu}\right)$ where the Lévy measure is concentrated on $(0, \infty)$ and such that

$$
v_{\mu}(\mathrm{d} x)=\frac{\mathrm{d} x}{x} \int_{0}^{\infty} e^{-x s} U_{\mu}(\mathrm{d} s)
$$

It is known that the class $T^{*}\left(\mathbb{R}_{+}\right)$is characterized by Wiener-Gamma representations, i.e., random integral representations with respect to the standard one-dimensional Gamma process (see [10], [9]). Specifically, a positive random variable $Y$ belongs to $T^{*}\left(\mathbb{R}_{+}\right)$if and only if there is a Borel function $h: \mathbb{R}_{+} \rightarrow \mathbb{R}_{+}$with

$$
\int_{0}^{\infty} \log (1+h(t)) \mathrm{d} t<\infty
$$

such that $Y \stackrel{\mathrm{d}}{=} Y^{h}$ has the Wiener-Gamma integral representation

$$
Y^{h} \stackrel{\mathscr{L}}{=} \int_{0}^{\infty} h(u) \mathrm{d} \gamma_{u}
$$


where $\left(\gamma_{t} ; t \geq 0\right)$ is the standard Gamma process with Lévy measure $v(\mathrm{~d} x)=e^{-x} \frac{\mathrm{d} x}{x}$. Moreover,

$$
\mathscr{C}_{Y^{h}}^{*}(z)=-\int_{0}^{\infty} \log \left(1+\frac{i z}{x}\right) U_{\mu}^{h}(\mathrm{~d} x)
$$

where $U_{\mu}^{h}$ denotes the image of Lebesgue measure on $(0, \infty)$ under the application $: s \rightarrow 1 / h(s)$. That is,

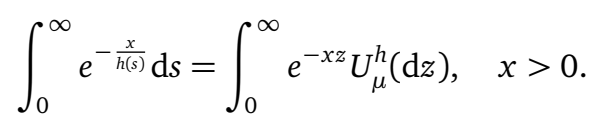

The function $h$ is called the Thorin function of $Y$ and is obtained as follows. Let $F_{U_{\mu}}(x)=\int_{0}^{x} U_{\mu}(\mathrm{d} y)$ for $x \geq 0$ and let $F_{U_{\mu}}^{-1}(s)$ be the right continuous inverse of $F_{U_{\mu}}^{-1}(s)$ in the sense of composition of functions, that is $F_{U_{\mu}}^{-1}(s)=\inf \left\{t>0 ; F_{U_{\mu}}(t) \geq s\right\}$ for $s \geq 0$. Then, $h(s)=1 / F_{U_{\mu}}^{-1}(s)$ for $s \geq 0$. Moreover we have the following alternative expression for the cumulant function of $\mu$

$$
\mathscr{C}_{\mu}^{*}(z)=-\int_{0}^{\infty} \mathrm{d} s \int_{0}^{\infty}\left(1-e^{i z x}\right) \frac{e^{-x / h(s)}}{x} \mathrm{~d} x .
$$

In the above equation, if $\left\{t>0 ; F_{U_{\mu}}(t) \geq s\right\}=\phi, x / h(s)=0$.

Remark 1.1. If $Y$ is a GGC random variable, we write $Y^{h}$ (respectively $\mu^{h}$ ) to indicate that it has the integral representation (6) and write $\mu^{h}=\mathscr{L}^{*}\left(Y^{h}\right)$. We have excluded from the above discussion the case of non-zero drift, which is easily incorporated by considering nonzero drift $c_{0}$ in the $*$-triplet $\left(c_{0}, 0, v_{\mu}\right)$.

Many well known distributions belong to $T^{*}\left(\mathbb{R}_{+}\right)$. The positive $\alpha$-stable distributions, $0<\alpha<1$, are GGC with $h(s)=\{s \theta \Gamma(\alpha+1)\}^{-\frac{1}{\alpha}}$ for a $\theta>0$. In particular, for the $1 / 2-$ stable distribution, $h(s)=4\left(s^{2} \pi\right)^{-1}$. First passage time distribution, Beta distribution of the second kind, lognormal and Pareto are also GGC, see [9].

As for distributions in $T^{*}(\mathbb{R})$, there is a another random integral representation approach recently presented in Barndorff-Nielsen et. al [1], who also considered the multivariate case. We recall that if $\left(X_{t} ; t \geq 0\right)$ is a $*$-Lévy process and $f:[a, b] \rightarrow \mathbb{R}$ is a continuous function defined on an interval $[a, b]$ in $[0, \infty)$, then the stochastic integral $\int_{[a, b]} f(t) d X_{t}$ may be defined as the limit in probability of approximating Riemann sums. Moreover, if $f$ is continuous function defined on $[0, \infty), \int_{[a, \infty)} f(t) d X_{t}$ may be as the limit in probability of $\int_{[a, b)} f(t) d X_{t}$ when $b \rightarrow \infty$. For stochastic integrals of nonrandom functions with respect to general additive processes we refer to Sato [14].

It is shown in [1], that for any $\mu$ in $I^{*}(\mathbb{R})$, the mapping $\Upsilon_{*}$ given by

$$
\Upsilon_{*}(\mu)=\mathscr{L}^{*}\left(\int_{0}^{1} \log \frac{1}{t} \mathrm{~d} X_{t}^{(\mu)}\right),
$$

is always defined, where $X_{t}^{(\mu)}$ is a Lévy process with $\mathscr{L}^{*}\left(X_{1}^{(\mu)}\right)=\mu$. Moreover $T^{*}(\mathbb{R})=\Upsilon_{*}\left(L^{*}(\mathbb{R})\right)$, where $L^{*}(\mathbb{R})$ is the class of $*$-selfdecomposable distributions in $\mathbb{R}: \mu \in L^{*}(\mathbb{R})$ if for any $b \in(0,1)$ there exists $\rho_{b} \in \mathscr{P}(\mathbb{R})$ such that $\hat{\mu}(z)=\hat{\mu}(b z) \hat{\rho_{b}}(z)$. Furthermore, it is shown in [1] that a random variable $Y$ belongs to $T^{*}(\mathbb{R})$ if and only if there exists $\mu \in I_{\log }^{*}(\mathbb{R})$ such that

$$
Y=\int_{0}^{\infty} e_{1}^{-1}(t) \mathrm{d} X_{t}^{(\mu)}
$$


where the function $e_{1}^{-1}(t)$ is the inverse of the incomplete gamma function $e_{1}(x)=\int_{x}^{\infty} e^{-s} s^{-1} \mathrm{~d} s$ and $X_{t}^{(\mu)}$ is a Lévy process with $\mathscr{L}^{*}\left(X_{1}^{(\mu)}\right)=\mu$.

In the study of relations between classical and free infinite divisibility, Bercovici and Pata [5] introduced a bijection $\Lambda$ between the set $I^{*}(\mathbb{R})$ of classical infinitely divisible laws and the set $I^{\boxplus}(\mathbb{R})$ of free infinitely divisible laws. A new approach to this bijection was recently proposed by BenaychGeorges [4] and Cabanal-Duvillard [8]. They construct random matrix ensembles associated to classical one-dimensional infinitely divisible laws whose empirical spectral laws converge to their corresponding free infinitely divisible laws under $\Lambda$. Recall that an ensemble of random matrices is a sequence $\left(M^{d}\right)_{d \geq 1}$ where for each $d \geq 1, M^{d}$ is a $d \times d$ matrix with random entries. The (random) spectral measure (or empirical spectral law) $\widehat{\mu}_{d}^{M^{d}}$ of $M^{d}$ is defined as the uniform distribution on the spectrum $\lambda_{1}^{M^{d}}, \ldots, \lambda_{d}^{M^{d}}$, that is, $\widehat{\mu}_{d}^{M^{d}}=d^{-1} \sum_{i=1}^{d} \delta_{\lambda_{i}^{M^{d}}}$. An ensemble $\left(M^{d}\right)_{d \geq 1}$ is a Random Matrix Model (RMM) for a probability measure $\mu$, if $\widehat{\mu}_{d}^{M^{d}}$ converges to $\mu$ weakly in probability as $d \rightarrow \infty$. It is shown in [4], [8] that for any $\mu \in I^{*}(\mathbb{R})$, there exists a random matrix model $\left(M^{d}\right)_{d \geq 1}$ for $\Lambda(\mu)$, which is constructed from $\mu$. These papers generalize the pioneering work by Wigner who connects Gaussian and semicircle laws throughout the Gaussian Unitary Ensemble of random matrices.

The purpose of this work is to study the free infinitely divisible laws (FGGC) corresponding to the image of $\Lambda$ of classical Generalized Gamma Convolutions and their corresponding random matrix models. We start in Section 2 by recalling facts and notation about the free cumulant function, the Bercovici-Pata bijection, free Lévy process and their random integrals. In Section 3 we prove a characterization of the free cumulant transform of a FGGC analogous to the classical cumulant transform (3). Furthermore, we derive free integral representations with respect to the free Gamma process and a Lévy process similar to (6) and (10), respectively. In Section 4 we construct random matrix models for FGGC. They are given as (classical) matrix random integrals of Wiener-Gamma type similar to (6), with respect to an appropriate (classical) matrix Gamma process. Finally, in Section 5 we point out some facts on nested subclasses of $\Lambda\left(T^{*}(\mathbb{R})\right.$ ) and their limits, analogous to the recent results for the classical convolution case study in Maejima and Sato [11].

\section{Preliminaries on Free infinite divisibility}

The Cauchy-Stieltjes transform of a probability measure $\mu$ on $\mathbb{R}$ is defined by

$$
G_{\mu}(z)=\int_{\mathbb{R}} \frac{1}{z-t} \mu(\mathrm{d} t), \quad z \in \mathbb{C}^{+} .
$$

The function $F_{\mu}(z)=1 / G_{\mu}(z)$ has right inverse $F_{\mu}^{-1}(z)$ on the region $\Gamma_{\eta, M}$ for some $M>0$ and $\eta>0$, where

$$
\Gamma_{\eta, M}:=\{z \in \mathbb{C}:|\operatorname{Re}(z)|<\eta \operatorname{Im}(z), \operatorname{Im}(z)>M\} ;
$$

(see Bercovici and Voiculescu [6]). Following Barndorff-Nielsen and Thorbjørnsen [3], the free cumulant transform $\mathscr{C}_{\mu}^{\boxplus}$ of $\mu$ is defined by

$$
\mathscr{C}_{\mu}^{\boxplus}(z)=z F_{\mu}^{-1}\left(z^{-1}\right)-1 \quad z^{-1} \in \Gamma_{\eta, M} .
$$

A probability measure $\mu$ on $\mathbb{R}$ is $\boxplus$-free infinitely divisible if and only if $\mathscr{C}_{\mu}^{\boxplus}(z)$ has an analytic continuation to $\mathbb{C}^{-}$. We denote by $I^{\boxplus}(\mathbb{R})$ the class of all free infinitely divisible distributions. In 
complete analogy to the classical case, the free Lévy-Khintchine characterization establishes that a probability measure $\mu$ belongs to $I^{\boxplus}(\mathbb{R})$ if only if

$$
\mathscr{C}_{\mu}^{\boxplus}(z)=\eta_{\mu} z+a_{\mu} z^{2}+\int_{\mathbb{R}}\left(\frac{1}{1-t z}-1-t z 1_{[-1,1]}(t)\right) v_{\mu}(\mathrm{d} t), \quad z \in \mathbb{C}^{-},
$$

where $a_{\mu} \in \mathbb{R}_{+}, \eta_{\mu} \in \mathbb{R}$ and the Lévy measure $v_{\mu}$ is a measure satisfying $v_{\mu}(\{0\})=0$ and $\int_{\mathbb{R}}\left(|x|^{2} \wedge\right.$ 1) $v_{\mu}(\mathrm{d} x)<\infty$. In this case, the $\boxplus$-triplet $\left(a_{\mu}, v_{\mu}, \eta_{\mu}\right)$ is uniquely determined by $\mu$ and is called the $\boxplus$-characteristic triplet or $\boxplus$-triplet for $\mu$, see [3] [6].

Bercovici and Pata [5] introduced a bijection $\Lambda$ between classical and free infinitely divisible distributions. It is such that if $\mu \in I^{*}(\mathbb{R})$ has $*$-characteristic triplet $\left(a_{\mu}, v_{\mu}, \eta_{\mu}\right)$, then $\Lambda(\mu)$ is the free infinitely divisible distribution with $\boxplus$-triplet $\left(a_{\mu}, v_{\mu}, \eta_{\mu}\right)$.

Remark 2.1. If $\mu \in I^{\boxplus}(\mathbb{R})$ and its Lévy measure $v_{\mu}$ satisfies $\int_{\mathbb{R}}|x| v_{\mu}(d x)<\infty$, then for $z \in \mathbb{C}^{-}$

$$
\begin{aligned}
\mathscr{C}_{\mu}^{\boxplus}(z) & =\eta_{\mu} z+a_{\mu} z^{2}+\int_{\mathbb{R}}\left(\frac{1}{1-t z}-1-t z 1_{[-1,1]}(t)\right) v_{\mu}(\mathrm{d} t) \\
& =\left(\eta_{\mu}-\int_{\{|x| \leq 1\}} x v(d x)\right) z+a_{\mu} z^{2}+\int_{\mathbb{R}}\left(\frac{1}{1-t z}-1\right) v_{\mu}(\mathrm{d} t) \\
& =\eta_{\mu}^{\prime} z+a_{\mu} z^{2}+\int_{\mathbb{R}}\left(\frac{1}{1-t z}-1\right) v_{\mu}(\mathrm{d} t),
\end{aligned}
$$

where $\eta_{\mu}^{\prime}=\eta_{\mu}-\int_{\{|x| \leq 1\}} x v_{\mu}(d x)$. We call this representation the drift type $\boxplus$-cumulant of $\mu \in I^{\boxplus}(\mathbb{R})$ and $\eta_{\mu}^{\prime}$ is the $\boxplus$-drift. By Bercovici-Pata bijection, if $\mu \in I^{*}(\mathbb{R})$ has $*$-drift type triplet $\left(a_{\mu}, v_{\mu}, \eta_{\mu}^{\prime}\right)$ then the $\boxplus$-drift type triplet of $\Lambda(\mu)$ is also $\left(a_{\mu}, v_{\mu}, \eta_{\mu}^{\prime}\right)$.

We summarize some properties of the Bercovici-Pata bijection in the following result (see [3], [5], [6]).

Proposition 2.2. The map $\Lambda: I^{*}(\mathbb{R}) \rightarrow I^{\boxplus}(\mathbb{R})$ has the following properties.

(1) $\Lambda(\mu * \rho)=\Lambda(\mu) \boxplus \Lambda(\rho)$ for any $\mu, \rho \in \mathscr{P}(\mathbb{R})$.

(2) Let $\delta_{a}$ be Dirac measure at $a$. $\Lambda\left(\delta_{a}\right)=\delta_{a}$ for $a \in \mathbb{R}$. So $\Lambda$ is preserved under affine transforms, i.e. $\Lambda\left(D_{c} \mu * \delta_{a}\right)=D_{c} \Lambda(\mu) \boxplus \delta_{a}$ for any $b>0$ and $a \in \mathbb{R}$ where $D_{c} \mu$ means the spectral distribution of the operator $c X$ with $\mu=\mathscr{L}(X)$.

(3) $\Lambda$ is a homeomorphism w.r.t. weak convergence i.e. $\mu_{n} \rightarrow \mu$ if and only if $\Lambda\left(\mu_{n}\right) \rightarrow \Lambda(\mu)$ in weak convergence.

For a classical random variable $X$ or a stochastic process $\left(X_{t}\right)$, we write $\Lambda(X)$ and $\Lambda\left(X_{t}\right)$ as a short notation for $\Lambda\left(\mathscr{L}^{*}(X)\right)$ and $\Lambda\left(\mathscr{L}^{*}\left(X_{t}\right)\right)$.

Barndorff-Nielsen and Thorbjørnsen [3] introduced free selfdecomposable distribution. A probability measure $\mu$ on $\mathbb{R}$ is free selfdecomposable ( $\boxplus$-selfdecomposable) if, for any $b \in(0,1)$, there exists $\rho_{b} \in \mathscr{P}(\mathbb{R})$ such that $\mu=D_{b} \mu \boxplus \rho_{b}$. We denote by $L^{\boxplus}(\mathbb{R})$ the class of all free selfdecomposable distributions on $\mathbb{R}$. We refer to Sakuma [15] for a detailed study of $\boxplus$-selfdecomposable distributions.

As in the classical case, free Lévy process and their free integrals can be considered with respect to the $\boxplus$-convolution. Given a free random variable $Z$, we denote by $\mathscr{L}^{\boxplus}(Z)$ its spectral distribution. Following [3], we say that a process $\left(Z_{t} ; t \geq 0\right)$ of selfadjoint operators affiliated with a 
$W^{*}$-probability space $(\mathscr{A}, \tau)$, is a free Lévy process (in law) if it satisfies the following four conditions:

(1) $Z_{0}=0$

(2) Whenever $n \in \mathbb{N}$ and $0 \leq t_{0}<t_{1}<\cdots t_{n}$, the increments

$$
Z_{t_{0}}, Z_{t_{1}}-Z_{t_{0}}, Z_{t_{2}}-Z_{t_{1}}, \cdots, Z_{t_{n}}-Z_{t_{n-1}}
$$

are freely independent random variables.

(3) For any $s, t$ in $[0, \infty), \mathscr{L}^{\boxplus}\left(Z_{s+t}-Z_{s}\right)$ does not depend on $\mathrm{s}$.

(4) For any $s \in[0, \infty), \mathscr{L}^{\boxplus}\left(Z_{s+t}-Z_{s}\right)$ converge weakly to $\delta_{0}$, as $t \rightarrow 0$.

For any compact interval $[a, b] \subset[0, \infty)$ and any continuous function $f:[a, b] \rightarrow \mathbb{R}$, the random integral $\int_{a}^{b} f(t) \mathrm{d} Z_{t}$ exists as the limit in probability of approximating Riemann sums. The following result summarizes the connection between classical and free random integrals, see [3].

Proposition 2.3. Let $\left(X_{t}\right)$ be a classical Lévy process and $\left(Z_{t}\right)$ be a free Lévy process with marginal distribution $\mu_{t}$ and $\Lambda\left(\mu_{t}\right)$, respectively. Then for any $[a, b] \subset[0, \infty)$ and any continuous function $f:[a, b] \rightarrow \mathbb{R}$, the laws $\mathscr{L}^{*}\left(\int_{a}^{b} f(t) d X_{t}\right)$ and $\mathscr{L}^{\boxplus}\left(\int_{a}^{b} f(t) d Z_{t}\right)$ are *-infinitely divisible and $\boxplus$ infinitely divisible, respectively. Moreover,

$$
\mathscr{L}^{\boxplus}\left(\int_{a}^{b} f(t) \mathrm{d} Z_{t}\right)=\Lambda\left(\mathscr{L}^{*}\left(\int_{a}^{b} f(t) \mathrm{d} X_{t}\right)\right) .
$$

In particular, if $Y$ is a free selfdecomposable random variable, there exists a free Lévy process $Z_{t}$ such that $\mathscr{L}\left(Z_{1}\right)=\mu, \int_{\mathbb{R} \backslash(-1,1)} \log (1+|t|) v_{\mu}(d t)<\infty$ and $\mathscr{L}^{\boxplus}(Y)=\int_{0}^{\infty} e^{-t} d Z_{t}$.

\section{Free Generalized Gamma Convolutions}

When $\gamma$ is the classical gamma distribution, we call $\Lambda(\gamma)$ the free gamma distribution. If $\left(\gamma_{t} ; t \geq 0\right)$ is the standard Gamma process, the free Lévy process $\left(\Lambda\left(\gamma_{t}\right) ; t \geq 0\right)$ is called the free standard Gamma process.

We say that a probability distribution $\lambda$ is Free Generalized Gamma Convolution (FGGC) (resp. Free Thorin) if there is a classical GGC (resp. Thorin) $\mu$ such that $\lambda=\Lambda(\mu)$. We denote by $T^{\boxplus}\left(\mathbb{R}_{+}\right)=\Lambda\left(T^{*}\left(\mathbb{R}_{+}\right)\right)$and $T^{\boxplus}(\mathbb{R})=\Lambda\left(T^{*}(\mathbb{R})\right)$ the classes of FGGC and Free Thorin class respectively. It follows trivially from Proposition $\left[2.2\right.$, that $T^{\boxplus}\left(\mathbb{R}_{+}\right)$is the smallest class that contains all free Gamma distributions and that is closed under $\boxplus$-convolution and convergence, while $T^{\boxplus}(\mathbb{R})$ is the smallest class on the real line $\mathbb{R}$ which contains $T^{\boxplus}\left(\mathbb{R}_{+}\right)$and is closed under convolution, convergence and reflection.

The following result is a characterization of the free cumulant transform of distributions in $T^{\boxplus}\left(\mathbb{R}_{+}\right)$ in terms of the Cauchy transform of the exponential distribution.

Theorem 3.1. A probability measure $\lambda$ in $\mathbb{R}_{+}$is FGGC without drift term if and only if there exists a Borel function $h: \mathbb{R}_{+} \rightarrow \mathbb{R}_{+}$satisfying (5) such that $\lambda$ has free cumulant transform

$$
\mathscr{C}_{\lambda}^{\boxplus}(z)=\int_{0}^{\infty} h(s) G_{E\left(\frac{1}{h(s)}\right)}\left(z^{-1}\right) \mathrm{d} s \quad z \in \mathbb{C}^{-},
$$


where $G_{E(a)}$ is the Cauchy transform of the exponential law with mean $1 / a$, i.e.

$$
G_{E(a)}(z)=\int_{0}^{\infty} \frac{a e^{-a x}}{z-x} \mathrm{~d} x \quad z \in \mathbb{C}^{+} .
$$

Alternatively, a probability measure $\lambda$ in $\mathbb{R}_{+}$is FGGC without drift term if and only if there is a Thorin measure $U_{\mu^{h}}$ such that

$$
\mathscr{C}_{\lambda}^{\boxplus}(z)=\int_{0}^{\infty} \frac{1}{s} G_{E(s)}\left(z^{-1}\right) U_{\mu}(\mathrm{d} s) \quad z \in \mathbb{C}^{-} .
$$

Proof. For any $t \geq 0$, the Lévy measure of $\left(\gamma_{t}\right)$ has finite first moment. We work with the drift type representation (12) with $\eta_{\mu}^{\prime}=a_{\mu}=0$. First, since $\left(\gamma_{t}\right)$ and $\left(\Lambda\left(\gamma_{t}\right)\right)$ have the same characteristic $*$ and $\boxplus$-triplet, from (12), the free cumulant transform of $\Lambda\left(\gamma_{t}\right)$ is obtained as

$$
\begin{aligned}
\mathscr{C}_{\Lambda\left(\gamma_{t}\right)}(z) & =t \int_{0}^{\infty}\left(\frac{1}{1-x z}-1\right) \frac{e^{-x}}{x} \mathrm{~d} x \\
& =t \int_{0}^{\infty} \frac{e^{-x}}{z^{-1}-x} \mathrm{~d} x \\
& =t G_{E(1)}\left(z^{-1}\right) \quad z \in \mathbb{C}^{-} .
\end{aligned}
$$

Next, by Remark 1.1 , a probability measure $\lambda$ without drift term belongs to $T^{\boxplus}\left(\mathbb{R}_{+}\right)$, if and only if there is a Thorin function $h$ such that $\lambda=\Lambda\left(\mu^{h}\right)$, where $\mu^{h}$ is in $T^{*}\left(\mathbb{R}_{+}\right)$with Thorin function and measure $h$ and $U_{h}$ respectively. Since $\mu^{h}$ and $\Lambda\left(\mu^{h}\right)$ have the same Lévy measure

$$
v_{\mu^{h}}(\mathrm{~d} x)=\frac{\mathrm{d} x}{x} \int_{0}^{\infty} e^{-x s} U_{\mu^{h}}(\mathrm{~d} s)
$$

from (12) and (17), the free cumulant transform of $\lambda$ is obtained as

$$
\begin{aligned}
\mathscr{C}_{\lambda}(z) & =\int_{0}^{\infty}\left(\frac{1}{1-x z}-1\right) \frac{\mathrm{d} x}{x} \int_{0}^{\infty} e^{-x s} U_{\mu^{h}}(\mathrm{~d} s) \mathrm{d} x \\
& =\int_{0}^{\infty} \frac{1}{s} G_{E(s)}\left(z^{-1}\right) U_{\mu}(\mathrm{d} s) \quad z \in \mathbb{C}^{-},
\end{aligned}
$$

which proves (16) and the if part of the second statement of theorem. For the converse, let $U_{h}$ be a Thorin measure and $\lambda$ be a probability measure such that (16) is satisfied. Let $v_{\mu^{h}}(\mathrm{~d} x)$ be the Lévy measure given by (18) and let $\mu^{h}$ be the corresponding measure in $T^{*}\left(\mathbb{R}_{+}\right)$. Then, from (19) and the uniqueness of the Lévy-Khintchine representation, $\lambda$ has Lévy measure $v_{\mu^{h}}(\mathrm{~d} x)$. Thus, by Bercovici-Para bijection $\lambda=\Lambda\left(\mu^{h}\right)$ and therefore $\lambda \in T^{\boxplus}\left(\mathbb{R}_{+}\right)$.

Finally, to prove the first statement of the theorem, we use (7) in (19), proceed as in (17) and by using (15) we obtain that

$$
\begin{aligned}
\mathscr{C}_{\lambda}(z) & =\int_{0}^{\infty}\left(\frac{1}{1-x z}-1\right) \frac{\mathrm{d} x}{x} \int_{0}^{\infty} e^{-x s} U_{\mu^{h}}(\mathrm{~d} s) \mathrm{d} x \\
& =\int_{0}^{\infty} h(s) G_{E x\left(\frac{1}{h(s)}\right)}\left(z^{-1}\right) \mathrm{d} s \quad z \in \mathbb{C}^{-} .
\end{aligned}
$$

Thus, (14) and (16) are equivalent. 
Using Propositions 2.2 and 2.3 , we can easily deduce integral representations for FGGC. First, if $Y^{h} \in T^{*}\left(\mathbb{R}_{+}\right)$has Wiener-Gamma representation (6), then

$$
\Lambda\left(Y^{h}\right)=\mathscr{L}^{\boxplus}\left(\int_{0}^{\infty} h(t) \mathrm{d} \Lambda\left(\gamma_{t}\right)\right) .
$$

Secondly, for any $\mu$ in $I^{\boxplus}(\mathbb{R})$, define the mapping $\Upsilon_{\boxplus}$ as

$$
\Upsilon_{\boxplus}(\mu)=\mathscr{L}^{\boxplus}\left(\int_{0}^{1} \log \frac{1}{t} \mathrm{~d} Z_{t}^{(\mu)}\right) .
$$

where $Z_{t}^{\mu}$ is free Lévy process with $\mathscr{L}^{\boxplus}\left(Z_{1}^{(\mu)}\right)=\mu$. Then it is easily seen that $\Lambda\left(\Upsilon_{*}(\mu)\right)=\Upsilon_{\boxplus}(\Lambda(\mu))$ and that $T^{\boxplus}(\mathbb{R})=\Upsilon_{\boxplus}\left(L^{\boxplus}(\mathbb{R})\right)$. Moreover,

$$
T^{\boxplus}(\mathbb{R})=\left\{\mathscr{L}^{\boxplus}\left(\int_{0}^{\infty} e_{1}^{-1}(t) \mathrm{d} Z_{t}^{(\mu)}\right): \mu \in \Lambda\left(I_{\log }^{*}(\mathbb{R})\right)\right\} .
$$

We now consider some examples of FGGC. A probability measure $\mu$ on $\mathbb{R}$ is called free stable $(\boxplus$-stable), if the class

$$
\{\psi(\mu): \psi \text { is an increasing affine transformation }\}
$$

is closed under the operation $\boxplus$. Let $S^{\boxplus}(\mathbb{R})$ denote the class of all free stable distributions on $\mathbb{R}$. The free domains of attractions of $S^{\boxplus}(\mathbb{R})$ were studied in [5]. As in the classical case, only the free Gaussian, the Cauchy and the free 1/2-stable have densities with closed form [5]. In the next example we further study the free $1 / 2$-stable, pointing out that it is also infinitely divisible and GGC in the classical sense.

Example 3.2. Let $\mu$ be the law of classical $\frac{1}{2}$-stable law (sometimes called Lévy distribution) with scale parameter $c$ and drift $c_{0} \geq 0$ (so its Lévy measure is $v(d r)=c r^{-3 / 2} d r$ ). It is easy to see that $\Lambda(\mu)$ has density

$$
g(x)=\frac{c}{\pi} \frac{\sqrt{\left(x-c_{0}\right)-\frac{c^{2}}{4}}}{\left(x-c_{0}\right)^{2}} \quad\left(x>\frac{c^{2}}{4}+c_{0}\right)
$$

with Laplace transform

$$
\mathbb{E}_{\Lambda(\mu)}[\exp (-r X)]=\frac{2}{\pi} \exp \left(-r\left(\frac{c^{2}}{4}+c_{0}\right)\right) \int_{0}^{\infty}(t+1)^{-2} t^{\frac{1}{2}} \exp \left(-\frac{r c^{2}}{4} t\right) \mathrm{d} t \quad r>0 .
$$

From this expression we deduce that $\Lambda(\mu)$ is the Beta distribution of the second kind $B_{2}\left(\frac{1}{2}, \frac{3}{2}\right)$. Bondesson [7, pp 59] proved that Beta distributions of second kind are GGC. Thus, $\Lambda(\mu)$ belongs to $T^{*}\left(\mathbb{R}_{+}\right)$ and $T^{\boxplus}\left(\mathbb{R}_{+}\right)$. It is an open problem whether free stable distributions other than free Cauchy and free $\frac{1}{2}$-stable are also infinitely divisible in the classical sense.

Example 3.3. We compute the free cumulant transform of four FGGC examples arising from classical GGC whose Thorin measures are considered in [9]. From these expressions their corresponding free cumulants are readily obtained. 
(1) Let $\mu$ be in $T^{\boxplus}\left(\mathbb{R}_{+}\right)$with the Thorin measure $U_{\mu}(d x)=\sum_{n=1}^{\infty} \delta_{\frac{\pi^{2}}{8}(2 n-1)^{2}}(d x)$. Then,

$$
\begin{aligned}
\mathscr{C}_{\mu}^{\boxplus}(z) & =\sum_{n=1}^{\infty} \frac{8}{\pi^{2}(2 n-1)^{2}} G_{E\left(\frac{\pi^{2}}{8}(2 n-1)^{2}\right)}\left(z^{-1}\right) \quad z \in \mathbb{C}^{-} \\
& =\sum_{k=1}^{\infty} k !\left(\sum_{n=1}^{\infty}\left\{\frac{8}{\pi^{2}(2 n-1)^{2}}\right\}\right) z^{k+1} \quad z \in \mathbb{C}^{-} .
\end{aligned}
$$

(2) Let $\mu$ be in $T^{\boxplus}\left(\mathbb{R}_{+}\right)$with the Thorin measure $U_{\mu}(d x)=\sum_{n=1}^{\infty} \delta_{\frac{\pi^{2} n^{2}}{2}}(d x)$. Then,

$$
\begin{aligned}
\mathscr{C}_{\mu}^{\boxplus}(z) & =\sum_{n=1}^{\infty} \frac{2}{\pi^{2} n^{2}} G_{E\left(\frac{\pi^{2} n^{2}}{2}\right)}\left(z^{-1}\right) \quad z \in \mathbb{C}^{-} \\
& =\sum_{k=1}^{\infty} k !\left(\frac{2}{\pi^{2}}\right)^{k+1}\left(\sum_{n=1}^{\infty} \frac{1}{n^{2(k+1)}}\right) z^{k+1} \quad z \in \mathbb{C}^{-} .
\end{aligned}
$$

(3) Let $\mu$ be in $T^{\boxplus}\left(\mathbb{R}_{+}\right)$with the Thorin measure $U_{\mu}(d x)=\frac{e^{-x u}}{\sqrt{u(2-u)}} 1_{(0,2)}(d x)$. Then,

$$
\mathscr{C}_{\mu}^{\boxplus}(z)=\sum_{k=1}^{\infty} \frac{1}{2^{2 k}(k !)^{2}} \int_{0}^{\infty} \frac{x^{2 k} e^{-x}}{z^{-1}-x} \mathrm{~d} x \quad z \in \mathbb{C}^{-} .
$$

(4) Let $\mu$ be in $T^{\boxplus}\left(\mathbb{R}_{+}\right)$with the Thorin measure $U_{\mu}(d x)=\frac{1}{\sqrt{u(2-u)}} 1_{(2, \infty)}(d x)$ Then,

$$
\mathscr{C}_{\mu}^{\boxplus}(z)=\int_{0}^{\infty} \mathrm{d} s \frac{1}{\sqrt{s(s+2)}} \int_{0}^{\infty} \frac{e^{-(2+s) x}}{z^{-1}-x} \mathrm{~d} x .
$$

\section{Random Matrix Models for Free GGC}

Let $\mathbb{M}_{d}=\mathbb{M}_{d}(\mathbb{C})$ denote the linear subspace of Hermitian matrices, with scalar product $\langle A, B\rangle \rightarrow$ $\operatorname{tr}\left(A B^{*}\right)$, for $A, B \in \mathbb{M}_{d}$ and tr denotes trace. By $\|M\|$ we denote the Euclidean norm. Let $\mathbb{M}_{d}^{+}$be the closed cone of nonnegative definite matrices in $\mathbb{M}_{d}$.

Let us first recall several facts on infinite divisibility of matrices taking values in the cone $\mathbb{M}_{d}^{+}$(see [2]). A $d \times d$ Hermitian random matrix $M$ is infinitely divisible in $\mathbb{M}_{d}^{+}$if and only if its cumulant transform $\mathscr{C}_{M}^{*}(A)=\log \mathbb{E}[\exp (i \operatorname{Tr}(A M))]$ is of the form

$$
\mathscr{C}_{M}^{*}(A)=i \operatorname{tr}\left(\Theta^{0} A\right)+\int_{\mathbb{M}_{d}^{+}}\left(e^{i \operatorname{tr}(X A)}-1\right) \rho(\mathrm{d} X), \quad A \in \mathbb{M}_{m}^{+},
$$

where $\Theta^{0} \in \mathbb{M}_{d}^{+}$is called the drift and the Lévy measure $\rho$ is such that $\rho\left(\mathbb{M}_{d} \backslash \mathbb{M}_{d}^{+}\right)=0$ and $\rho$ has order of singularity

$$
\left.\int_{\mathbb{M}_{d}^{+}} \min (1,\|X\|)\right) \rho(\mathrm{d} X)<\infty .
$$

Moreover, the Laplace transform of $M$ is given by

$$
\mathbb{E}[\exp (-\operatorname{tr}(M A))]=\exp \left\{-\operatorname{tr}\left(\Theta^{0} A\right)-\int_{\mathbb{M}_{d}^{+}}\left(1-e^{-\operatorname{tr}(X A)}\right) \rho(\mathrm{d} X)\right\} .
$$


If $M$ is an infinitely divisible matrix in $\mathbb{M}_{d}^{+}$, the associated matrix Lévy process $\left\{M_{t}\right\}_{t \geq 0}$ is called a matrix subordinator. It is $\mathbb{M}_{d}^{+}$-increasing in the sense that for all $0 \leq s<t, M_{t}-M_{s} \in \mathbb{M}_{d}^{+}$with probability one.

The matrix valued random integral

$$
N=\int_{0}^{\infty} f(t) \mathrm{d} M_{t}
$$

of a non-random real valued function $f$ is defined in the sense of integrals with respect to scattered random measures, see [12], [14]. When definable, it is a $d \times d$ infinitely divisible random matrix with cumulant transform

$$
\mathscr{C}_{N}^{*}(A)=\int_{0}^{\infty} \mathscr{C}_{M}^{*}(f(t) A) \mathrm{d} t .
$$

Of special interest in this work is the Gamma type matrix subordinator $\Gamma=\left\{\Gamma_{t}^{d}\right\}_{t \geq 0}$ corresponding to the Lévy measure

$$
\rho_{d}^{g}(\mathrm{~d} X)=\frac{\exp (-\|X\|)}{\|X\|} \widetilde{\omega}_{d}(\mathrm{~d} X)
$$

where $\widetilde{\omega}_{d} / d(E)=\int_{0}^{\infty} d r \int_{\mathbb{S}_{d}} \omega_{d}(d V) 1_{E}(r V) . \omega_{d}$ is the (probability) measure on $\mathbb{S}_{d}^{+}=\left\{A \in \mathbb{M}_{d}^{+}\right.$ $;\|A\|=1\}$ induced by the transformation $u \rightarrow V=u u^{*}$, where the column random vector $u$ is uniformly distributed on the unit sphere of $\mathbb{C}^{d}$. The Lévy measure $\rho_{d}^{g}$ has the polar decomposition

$$
\rho_{d}^{g}(E)=d \int_{\mathbb{S}_{d}^{+}} \omega_{d}(\mathrm{~d} V) \int_{0}^{\infty} 1_{E}(r V) \frac{e^{-r}}{r} \mathrm{~d} r .
$$

We observe that $\rho_{d}^{g}$ has support on the subset of rank one matrices in $\mathbb{M}_{d}^{+}$. The case $d=1$ corresponds to the Lévy measure of the one dimensional gamma process. The corresponding matrix random integral $\int_{0}^{\infty} h(t) \mathrm{d} \Gamma_{t}^{d}$ is called the matrix Wiener-Gamma integral and is defined for Borel functions $h: \mathbb{R}_{+} \rightarrow \mathbb{R}_{+}$satisfying (5).

The following is the main result of this section. It gives a RMM for FGGC on $\mathbb{R}_{+}$, where the RMM is given by matrix Wiener-Gamma type integrals, which are GGC matrix extensions of the one-dimensional case.

Theorem 4.1. Let $\mu^{h}$ be a classical GGC on $\mathbb{R}_{+}$given by the Wiener-Gamma integral

$$
\mu^{h}=\mathscr{L}\left(\int_{0}^{\infty} h(t) \mathrm{d} \gamma_{t}\right)
$$

The free GGC $\Lambda\left(\mu^{h}\right)$ has a RMM given by the ensemble of infinitely divisible matrix Wiener-Gamma integrals

$$
\left(M_{h}^{d}=\int_{0}^{\infty} h(t) \mathrm{d} \Gamma_{t}^{d}\right)_{d \geq 1},
$$

where for each $d \geq 1,\left\{\Gamma_{t}^{d}\right\}_{t \geq 0}$ is the Gamma type matrix subordinator associated to the Lévy measure $\rho_{d}^{g}$ given by (26).

Proof. We shall use Theorem 6.1 in [4], which establishes that for any $\mu \in I^{*}(\mathbb{R})$, there is an ensemble of random matrices $\left(M_{d}\right)_{d \geq 1}$ such that the spectral distribution of $M_{d}$ converges in probability to $\Lambda(\mu)$. Moreover, from Theorem 3.1 in [4], for each $d \geq 1$, the Fourier transform of the random matrix $M_{d}$ is given by the expression

$$
\mathbb{E}\left[\exp \left(i \operatorname{Tr}\left(A M_{d}\right)\right]=\exp \left\{\mathbb{E}_{u}\left(d \times \mathscr{C}_{\mu}^{*}(\langle u, A u\rangle)\right\}, \quad A \in \mathbb{M}_{m},\right.\right.
$$


where $u=\left(u_{1}, \ldots, u_{d}\right)^{t}$ is a uniformly distributed random vector on the unit sphere of $\mathbb{C}^{d}$ and $\mathscr{C}_{\mu}^{*}$ is the cumulant function (Lévy exponent) of $\mu$. We will show that when $\mu^{h}$ is a classical GGC, the random matrices $\left(M_{h}^{d}\right)_{d \geq 1}$ given by (28) have the same laws as $\left(M_{d}\right)_{d \geq 1}$ with Fourier transform (29), where $\mathscr{C}_{\mu}^{*}$ is the cumulant transform $\mathscr{C}_{\mu^{h}}^{*}$ of $\mu^{h}$. This will prove the theorem.

First, let $\mu$ be the one dimensional standard Gamma distribution, $d \geq 1$ be fixed and $u=$ $\left(u_{1}, \ldots, u_{d}\right)^{t}$ be a uniformly distributed random vector on the unit sphere of $\mathbb{C}^{d}$. Let $\Gamma_{1}^{d}$ be the Gamma type matrix subordinator at $t=1$ corresponding to the Lévy measure (26). We will show that

$$
\mathbb{E}\left[\exp \left(-\operatorname{Tr}\left(A \Gamma_{1}^{d}\right)\right)\right]=\exp \left\{-d \mathbb{E}_{u}\left[\int_{0}^{\infty}\left(1-e^{-\langle u, A u\rangle x}\right) \frac{e^{-x}}{x} \mathrm{~d} x\right]\right\} .
$$

Then, writing $V=u u^{*}$ and using the polar decomposition (27) we have

$$
\begin{aligned}
\mathbb{E}\left[\exp \left(-\operatorname{Tr}\left(A \Gamma_{1}^{d}\right)\right)\right] & =\exp \left\{-\int_{\mathbb{M}_{d}^{+}}\left(1-e^{-\operatorname{Tr}(A X)}\right) \frac{e^{-\|X\|}}{\|X\|} \widetilde{\omega}_{d}(\mathrm{~d} X)\right\} \\
& =\exp \left\{-d \int_{\mathbb{S}_{d}^{+}} \omega_{d}(\mathrm{~d} V) \int_{0}^{\infty}\left(1-e^{-\operatorname{Tr}(V A) x}\right) \frac{e^{-x}}{x} \mathrm{~d} x\right\} \\
& =\exp \left\{-d \mathbb{E}_{V}\left[\int_{0}^{\infty}\left(1-e^{-\operatorname{Tr}(V A) x}\right) \frac{e^{-x}}{x} \mathrm{~d} x\right]\right\} \\
& =\exp \left\{-d \mathbb{E}_{u}\left[\int_{0}^{\infty}\left(1-e^{-\operatorname{Tr}\left(u u^{*} A\right) x}\right) \frac{e^{-x}}{x} \mathrm{~d} x\right]\right\} .
\end{aligned}
$$

Second, let $\left(\mathbb{P}_{d}^{\mu^{h}}\right)_{d \geq 1}$ be the matrix distributions of the random matrices ensemble given by (28), where $\mu^{h}$ is a classical one dimensional GGC with Thorin function $h$. Using (25), (31) and (27), we have that

$$
\begin{aligned}
\mathbb{E}_{\mathbb{P}_{d}^{\mu^{h}}}\left[\exp \left(-\operatorname{Tr}\left(A M_{h}^{d}\right)\right)\right] & =\exp \left\{-\int_{0}^{\infty} \mathrm{d} s \int_{\mathbb{M}_{d}^{+}}\left(1-e^{-\operatorname{Tr}(A X) h(s)}\right) \frac{e^{-\|X\|}}{\|X\|} \widetilde{\omega}_{d}(\mathrm{~d} X)\right\} \\
& \exp \left\{-d \int_{0}^{\infty} \mathrm{d} s \int_{\mathbb{S}_{d}^{+}} \omega_{d}(\mathrm{~d} V) \int_{0}^{\infty}\left(1-e^{-\operatorname{Tr}(V A) h(s) x}\right) \frac{e^{-x}}{x} \mathrm{~d} x\right\} \\
& =\exp \left\{-d \mathbb{E}_{u} \int_{0}^{\infty} \mathrm{d} s \int_{0}^{\infty}\left(1-e^{-\operatorname{Tr}\left(u u^{*} A\right) x}\right) \frac{e^{-x / h(s)}}{x} \mathrm{~d} x\right\}
\end{aligned}
$$

From this Laplace transform and (8), we get (29).

Remark 4.2. If $\mu \in T^{*}\left(\mathbb{R}_{+}\right)$and without drift, then $\Lambda(\mu)$ is concentrated on $\mathbb{R}_{+}$. This follows trivially from the above construction of the RMM. As pointed out by the referee, this fact also follows from the well known equivalence

$$
v_{n}^{* n} \rightarrow_{n \rightarrow \infty} \mu \Longleftrightarrow v_{n}^{\boxplus n} \rightarrow_{n \rightarrow \infty} \Lambda(\mu) .
$$

Similar to the above theorem, we can construct RMM for GGC on $\mathbb{R}$, where the RMM is given by matrix random integrals similar to the one dimensional representation (10). 
Theorem 4.3. Let $\mu_{1}$ be in $T^{*}(\mathbb{R})$ given by the random integral representation

$$
\mu_{1}=\mathscr{L}\left(\int_{0}^{\infty} e_{1}^{-1}(t) \mathrm{d} X_{t}^{(\mu)}\right),
$$

for $\mu \in I_{\log }^{*}(\mathbb{R})$ and where $X_{t}^{(\mu)}$ is a Lévy process such that $\mathscr{L}\left(X_{1}^{(\mu)}\right)=\mu$. The free $G G C \Lambda\left(\mu_{1}\right)$ has a $R M M$ given by the ensemble of infinitely divisible matrix random integrals

$$
\left(M_{h}^{d}=\int_{0}^{\infty} e_{1}^{-1}(t) \mathrm{d} R_{t}^{d}\right)_{d \geq 1},
$$

where for each $d \geq 1,\left\{R_{t}^{d}\right\}_{t \geq 0}$ is a matrix valued Lévy process with Lévy measure $v_{d}$ given by

$$
v_{d}(E)=\int_{\mathbb{S}_{d}^{+}} \omega_{d}(\mathrm{~d} V) \int_{0}^{\infty} 1_{E}(r V) v(\mathrm{~d} r),
$$

with $\omega_{\mathrm{d}}$ as in (26) and $v$ is the Lévy measure of $\mu$.

\section{Inheritance of nested subclasses of FGGC and its limit class under $\Lambda$}

Maejima and Sato [11] proved that nested subclasses of classical Thorin distributions are characterized by limit theorem and proved that its limit class is the closure of the class of classic stable distributions $S^{*}(\mathbb{R})$, which is taken under $*$-convolution and weak convergence. We now point out a similar result for free Thorin distributions. The free selfdecomposable case was recently considered by Sakuma [15].

We define subclasses of $T^{\boxplus}(\mathbb{R})$ as follows. Let $\Psi=\int_{0}^{\infty} e_{1}^{-1}(t) \mathrm{d} Z_{t}^{(\mu)}$ be the free integral considered in $(20)$ and $I_{\text {logm }}^{*}(\mathbb{R})=\left\{\mu \in I^{*}(\mathbb{R}): \int_{\mathbb{R}}\left(\log ^{+}|x|\right)^{m} \mu(\mathrm{d} x)<\infty\right\}$.

(1) For $m=1,2$,...let $T_{m}^{\boxplus}(\mathbb{R})=\Lambda\left(\Psi\left(I_{\log ^{m+1}}^{*}(\mathbb{R})\right)\right)$ and $T_{\infty}^{\boxplus}(\mathbb{R})=\cap_{m=1}^{\infty} T_{m}^{\boxplus}(\mathbb{R})$.

(2) $\mu \in L_{m}^{\boxplus}(\mathbb{R})$ if, for any $c \in(0,1)$, there exists $\rho_{c} \in L_{m-1}^{\boxplus}(\mathbb{R})$ such that $\mu=D_{c} \mu \boxplus \rho_{c}$. We also define $L_{\infty}^{\boxplus}(\mathbb{R})=\cap_{m=0}^{\infty} L_{m}^{\boxplus}(\mathbb{R})$. It was proved in [15] that $L_{m}^{\boxplus}(\mathbb{R})$ is $\boxplus$-c.c.s.s. and $L_{\infty}^{\boxplus}=\overline{S^{\boxplus}(\mathbb{R})}$.

The following concept was introduced in the sense of classical convolution in [11].

Definition 5.1. A class $M$ of distributions on $\mathbb{R}$ is said to be * (resp. $\boxplus$ )-completely closed in the strong sense (*-c.c.s.s. (resp. $\boxplus$-c.c.s.s.)), if $M \subset I^{*}(\mathbb{R})$ (resp. $M \subset I^{\boxplus}(\mathbb{R})$ ) and if the following are satisfied.

(1) It is closed under * (resp. 田)-convolution.

(2) It is closed under weak convergence.

(3) If $\mu \in M$, then $D_{c} \mu * \delta_{b} \in M$ (resp. $D_{c} \mu \boxplus \delta_{b} \in M$ ) for any $c>0$ and $b \in \mathbb{R}$.

(4) $\mu \in M$ implies $\mu^{s *} \in M$ (resp. $\mu^{s \boxplus} \in M$ ) for any $s>0$, where $\mu^{s *}$ is the distribution with the cumulant $s C_{\mu}(z)$ (resp. $\mu^{s \boxplus}$ is the distribution with the free cumulant $s \mathscr{C}_{\mu}^{\boxplus}(z)$ ).

The closure is taken under $\boxplus$-convolution and weak convergence.

The following result gives the preservation of classical completely closed in the strong sense class under the Bercovici-Pata bijection.

Lemma 5.2. If $M$ is *-c.c.s.s., then $\Lambda(M)$ is $\boxplus$-c.c.s.s.. 
Proof. (1) and (2) in the above definition follow from Proposition 2.2. If $\mu \in \Lambda(M)$, then $\Lambda^{-1}\left(D_{c} \mu \boxplus\right.$ $\left.\delta_{b}\right)=D_{c} \Lambda^{-1}(\mu) \delta_{b} \in M$. So $D_{c} \mu \boxplus \delta_{b} \in \Lambda(M)$ and (3) holds. Finally, (4) holds from the classical and free Lévy Khintchine formulas.

From the above lemma and Proposition 2.3, we immediately obtain the following relationships.

Lemma 5.3. Fix $0<a<\infty$. Suppose $f$ is continuous on $(0, a)$ and $\int_{0}^{a} f(s) d s \neq 0$. Let $\left\{Z_{t}\right\}$ be $a$ free Lévy process with distribution $\mu$. Define the mapping

$$
\Phi_{f}^{\boxplus}(\mu)=\mathscr{L}^{\boxplus}\left(\int_{0}^{a} f(s) \mathrm{d} Z_{s}^{(\mu)}\right) .
$$

Then the following are true

(1) If $M$ is $\boxplus$-c.c.s.s., then $\Phi_{f}^{\boxplus}(M) \subset M$.

(2) If $M$ is $\boxplus$-c.c.s.s., then $\Phi_{f}^{\boxplus}(M)$ is also $\boxplus$-c.c.s.s.

Theorem 5.4.

$$
T_{\infty}^{\boxplus}(\mathbb{R})=L_{\infty}^{\boxplus}(\mathbb{R})=\overline{S^{\boxplus}(\mathbb{R})} .
$$

Proof. From $T_{m}^{\boxplus}(\mathbb{R})=\Upsilon_{\boxplus}^{m+1}\left(L_{m}^{\boxplus}(\mathbb{R})\right) \subset L_{m}^{\boxplus}(\mathbb{R})$, we have

$$
T_{\infty}^{\boxplus}(\mathbb{R}) \subset L_{\infty}^{\boxplus}(\mathbb{R}) .
$$

Since $T_{m}^{*}(\mathbb{R})$ is $*$-c.c.s.s., then $T_{m}^{\boxplus}(\mathbb{R})$ is $\boxplus$-c.c.s.s. It is clear that $T_{m}^{\boxplus}(\mathbb{R})=\Upsilon_{\boxplus}^{m+1}\left(L_{m}^{\boxplus}(\mathbb{R})\right) \subset$ $\Upsilon_{\boxplus}^{m+1}\left(S^{\boxplus}(\mathbb{R})\right)=S^{\boxplus}(\mathbb{R})$. Next, since $T_{m}^{\boxplus}(\mathbb{R})$ is $\boxplus$-c.c.s.s., $T_{m}^{\boxplus}(\mathbb{R}) \subset \overline{S^{\boxplus}(\mathbb{R})}$ and therefore,

$$
T_{\infty}^{\boxplus} \subset \overline{S^{\boxplus}(\mathbb{R})}=L_{\infty}^{\boxplus}(\mathbb{R}) .
$$

Then (33) and (34) yield

$$
T_{\infty}^{\boxplus}(\mathbb{R})=\overline{S^{\boxplus}(\mathbb{R})}=L_{\infty}^{\boxplus}(\mathbb{R}) .
$$

\section{Acknowledgment.}

The authors would like to thank the referee for a very careful reading of the manuscript and for the valuable suggestions and comments.

\section{References}

[1] O. E. Barndorff-Nielsen, M. Maejima and K.I. Sato, Some classes of multivariate infinitely divisible distributions admitting stochastic integral representations, Bernoulli, 12 (2006), 1-33. MR2202318

[2] O. E. Barndorff-Nielsen and V. Pérez-Abreu, Matrix subordinators and related upsilon transformations. Theory Probab. Appl., 52 (2008), 1-23 MR2354571

[3] O. E. Barndorff-Nielsen and S. Thorbjørnsen, Classical and free infinite divisibility and Lévy processes, in Quantum independent increment processes. II, 33-159, Lecture Notes in Math., 1866, Springer, Berlin. MR2213448 
[4] F. Benaych-Georges, Classical and free infinitely divisible distributions and random matrices, Ann. Probab., 33 (2005), 1134-1170. MR2135315

[5] H. Bercovici and V. Pata, Stable laws and domains of attraction in free probability theory, Ann. of Math., 149 (1999), 1023-1060. MR1709310

[6] H. Bercovici and D. Voiculescu. Free convolution of measures with unbounded support. Indiana Univ. Math. J., 42 (1993), 733-773. MR1254116

[7] L. Bondesson, Generalized gamma convolutions and related classes of distributions and densities, Lecture Notes in Statist., 76, Springer, New York, 1992. MR1224674

[8] T. Cabanal-Duvillard, A matrix representation of the Bercovici-Pata bijection, Electron. $J$. Probab., 10 (2005), 632-661 (electronic). MR2147320

[9] L. F. James, B. Roynette and M. Yor, Generalized gamma convolutions, Dirichlet means, Thorin measures, with explicit examples. arXiv:0708.3932V1 [math.PR] 29 Aug 2007.

[10] A. Lijoi and E. Regazzini, Means of a Dirichlet process and multiple hypergeometric functions, Ann. Probab., 32 (2004), 1469-1495. MR2060305

[11] M. Maejima and K.I. Sato, The Limits of nested subclasses of several classes of infinitely divisible distributions are identical with the closure of the class of stable distributions, to appear in Probab. Theory Related Fields.

[12] B. S. Rajput and J. Rosiński, Spectral representations of infinitely divisible processes, Probab. Theory Related Fields 82 (1989), 451-487. MR1001524

[13] K.I. Sato, Lévy processes and infinitely divisible distributions, Cambridge Univ. Press, Cambridge, 1999. MR1739520

[14] K.I. Sato, Additive processes and stochastic integrals, Illinois J. Math., 50 (2006), 825-851 (electronic). MR2247848

[15] N. Sakuma, Characterizations of the class of free self decomposable distributions and its subclasses, to appear in Inf. Dim. Anal. Quantum Probab..

[16] O. Thorin, On the infinite divisibility of the Pareto distribution, Scand. Actuar. J., 1977, 3140. MR0431333

[17] O. Thorin, On the infinite divisibility of the lognormal distribution, Scand. Actuar. J., 1977, 121-148. MR0552135

[18] O. Thorin, An extension of the notion of a generalized $\Gamma$-convolution, Scand. Actuar. J., 1978, 141-149. MR0514310 